\title{
S41. Biological and clinical changes in the course of mental disorders
}

\section{BIOLOGICAL CHANGES IN THE TREATMENT OF MENTAL DISORDERS \\ S. Kasper. G. Höflich, P. Danos, H.-J. Möller \\ University Hospital for Psychiatry, Department of General Psychiatry \\ A-1090 VIENNA, Währinger Gürtel 18-20, Austria}

Although there is a number of biological abnormalities of mental disorders reported in the literature, there is an obvious lack of data related to treatment procedures as well as in the natural course of the illness. We have undertaken different efforts to document treatment related changes in pharmacological (antidepressant therapy) and in nonpharmacological treatment procedures (electroconvulsive treatment, sleep deprivation). Fenfluramin induced prolactin and cortisol responses were not related to treatment outcomes when patients were treated with either fluvoxamine or maprotiline. However, pretreatment fenfluramine-induced prolactin values were negatively correlated with the response to the serotonergic compound fluvoxamine. Neuroendocrinological changes in the course of electroconvulsive treatment (ECT) were related to response in therapy refractory depressed patients; e.g. lower ECT-stimulated prolactin values were found in patients responding to ECT. Hormonal changes in sleep deprivation indicated that higher night time body temperature during the sleep deprivation procedure was found in patients responding to this procedure. These and further findings of our group indicate that different biological measurements should be obtained before as well as during the treatment course in mental disorders. It seems likely that they will allow us to monitor and probably predict short- and longterm outcome and would give us a rational basis for biologically oriented treatment approaches.
HORMONAL CHANGES IN THE COURSE OF DEPRESSION M. Ansseau. W. Pitchot, P. Papart, A. Gonzalez Moreno, S. Kjiri. J. J. Legros

Psychiatric Unit, C.H.U. du Sart Tilman, B-4000 Liège, Belgium

Major depression is accompanied by various hormonal disturbances. Moreover, neuroendocrine strategy may provide an indirect of central neurotransmission, which can be of particular interest in the diagnosis and treatment of depressive illness. In this presentation, we will briefly review our work concerning the clinical usefulness of 3 selective pharmacological challenges : the growth hormone (GH) response to iv clonidine $0.15 \mathrm{mg}$, an $\alpha 2$ noradrenergic agonist, and sc apomorphine $0.5 \mathrm{mg}$, a dopaminergic agonist, and the prolactin response to iv flesinoxan $1 \mathrm{mg}$, a 5-HT1A agonist.

The GH responses after clonidine were blunted (peak $<5 \mathrm{ng} / \mathrm{ml}$ ) in $80 \%$ of major depressive patients and negatively correlated with the MMPI depression, psychasthenia and social introversion subscales and positively correlated with the hypomania subscale. A blunted response was not associated with a higher level of life-events preceding hospitalization or with a history of suicide attempts. A normal response to clonidine was predictive of a better outcome with serotonergic antidepressants.

The $\mathrm{GH}$ responses to apomorphine were also significantly blunted in major depressive patients and positively correlated with the social introversion and the anxiety MMPI subscales. A blunted response was associated with a higher level of life-events preceding hospitalization, with a positive history of suicide attempts, and with more retardation. Whereas a normal response was predictive of a preferential outcome with serotonergic antidepressants, a blunted response was associated with a better outcome with catecholaminergic antidepressants.

The prolactin response to flesinoxan was significantly blunted in major depressive patients. Patients with a positive history of suicide attempts were characterized by lower cortisol responses as compared to patients with negative history. 
THE IMPACT OF IMPROVING COMPLIANCE ON THE COURSE OF SCHIZOPHRENIA

W.P.Hornung ${ }^{1}$, G. Buchkremer

'Psychiatric Department of the University of Münster, Germany, ${ }^{2}$ Psychiatric Department of the University of Tübingen, Germany

Medication noncompliance is a frequent reason for relapse in schizophrenic psychosis. Therefore, good compliance can be regarded as one condition for an efficacious prevention of repeated relapses. On the other hand compliance alone is not sufficient to ensure the non-occurrence of psychotic symptoms (HOGATAY et a!. 1986). Nevertheless, a current 40 to 50 percent rate of schizophrenic patients discontinuing their neuroleptics is not at all desirable and has to be lowered. Because of the "yes-nofallacy" (DIRKS and KINSMAN 1982) of compliance, need-adapted approaches of prophylactic interventions must be developed.

At the Psychiatric Department of the University of Münster, Germany, we carried out a prospective intervention study to evaluate a psychoeducational-psychotherapeutic training program for chronic schizophrenic patients and their relatives. The main objective of the trial was to reduce schizophrenic rehospitalization rates by improving medication compliance and problem solving skills.

The study comprised 191 mostly chronic DSM-III-R schizophrenic outpatients, 58 male, with a medium age of $31.3(+/-10.4) .71 \%$ of them showed a good compliance.

The paper presents data on the course of illness of the study patients within a one-and two-year follow-up after end of therapy. The results of our study will be discussed in detail.

\section{THE DETECTION OF RELAPSES IN TREATMENT OF} ALCOHOLISM

S.Borg, O Beck, A Helander, A Voltaire, AM Högström-Brandt. Karolinska Institute, Dept of Neuroscience, St Göran's Hospital and Dept of Clinical Pharmacology, Karolinska Hospital, Stockholm, Sweden.

In recent years the interest of the dynamics of relapse has increased. To study the relapse process in detail two objective markers were used to follow male alcohol dependent patients prospectively during a 6 months abstinence oriented program. Clinical items were registered three times per week. Carbohydrate deficient transferrin - CDT - in serum once a week and 5-hydroxytryptophol - 5HTOL - in urine once a day.

CDT has been shown to be a highly specific and sensitive marker for regular high alcohol consumption. Other drugs and major illnesses do not influence the CDT-levels and false positives has been shown to be less than $1-3 \%$. 5 HTOL is also a very sensitive and specific marker but only for recent alcohol intake, $<24$ hours. All patients showed signs of alcohol consumption during the observation period, while only a minority reported intake of alcohol. By using individual cut off levels for CDT the number of detected relapses could be increased. A considerable portion of the patients were able to drink in a controlled way. The clinical profile showed differences in those who reported relapses compared to non reporting patients.
THEIMPACT OF BRAIN ATROPHY AND COGNITIVE DEFICITS ON THE COURSE OF ALCOHOLISM

K.Mann K. Ackermann, A. Günther, M. Jung, D. Petersen', P. Wakat ${ }^{1}$

'Dept. of Psychiatry and Dept. of Neurology.

University of Tübingen Medical School, Osianderstr. 22, 72076 Tübingen

Alcohol dependent patients develop brain damage and cognitive deficits in the course of their disease. Both phenomena are reversible once abstinence is maintained. Their impact on longterm treatment outcome has not been studied systematically thus far.

We conducted and controlled study of 106 alcoholic patients (49 males, 57 females) and normal controls matched for age, gender and education. At the beginning and at the end of a six week inpatient treatment, patients underwent CT scans and were investigated neuropsychologically. Tests were repeated after six months at midterm of the outpatient part of treatment and again 12 months later, half a year after the end of treatment. Controls were tested twice.

So far all male patients were evaluated. They exhibited significant brain atrophy and cognitive deficits. Both were partially reversible during treatment. Neither had any influence on relapse rates, which was true for depressiveness, also. Results of the female population will be reported, as well as the influence of comorbidity and social support on the prognosis of the whole sample. 